\title{
Review and Further Prospects of Plant Disease Detection Using Machine Learning
}

\author{
Vempati Ramsanthosh, Anati Sai Laxmi, Chepuri Sai Abhinay, Vadepally Santosh, Vybhav Kothareddy, \\ Shivali Chopra \\ Department of Computer Science \& Engineering, Lovely Professional University, Punjab, India
}

\begin{abstract}
Identifying of the plant diseases is essential in prevention of yield and volume losses in agriculture Product. Studies of plant diseases mean studies of visually observable patterns on the plant. Health surveillance and detecting diseases in plants is essential for sustainable development agriculture. It is very difficult to monitor plant diseases manually. It requires a lot of experiences in work, expertise in these field plant diseases and also requires excessive processing time. Therefore; image processing is used to detect plant diseases. Disease detection includes steps such as acquisition, image Pre-processing, image segmentation, feature extraction and Classification. We describe these methods for the detection of plant diseases on the basis of their leaf images; automatic detection of plant disease is done by the image processing and machine learning. The different leaf images of plant disease are collected and feature extracted of the various machine learning methods.
\end{abstract}

Article Info

Volume 7, Issue 3

Page Number : 105-115

Publication Issue :

May-June-2021

\section{Article History}

Accepted : 08 May 2021

Published : 14 May 2021

Keywords : Plant diseases detection, image acquisition, image segmentation, features extraction.

\section{INTRODUCTION}

"The agriculture is primarily division to produces food resources". Highly economy depends on the agriculture. The plant disease occurs from that we use digital technologies. Now days most of the agriculture helps centres and many farmers are using different types of technologies to improve production in agriculture. From identifying the plant diseases we are using image pre-processing techniques [11].

By the image of plant diseases or initially spotted on the leaves of the plant [21]. By the image processing of image we can detect the disease and we can prevent from the particular disease. Texture statistics of the extracted region of the image segment is finally completed and preventing by providing according to analysis [7].

In most of the cases the symptoms are shows on the leave, stem and fruit [7]. We can capture of the image by camera or phone. The images will identity by an algorithm [9]. The analysis of image is done by an algorithm that identifies the disease and remedies/ solution to defend the mechanism of the disease and improve the growth of the plant. Image processing technique use's to identify the image of the affected 
region of the leaf is determined by the pattern, edge of the colour of the image [7] [19].

"The image segmentation is a procedure of image divide into parts of collective image segments [13]". Simplify the represent of the image is deeper and simple to analyze. The currently there many different ways of image segmentation, thresholding is the advance used to create binary image [17]. The segmentation process is depend on the variations of the image might be colour, boundaries or edges and segments of the image [13] [16].

The automatic detection of plant disease, machine learning algorithms is used in many ways [4]. It is used widely in the plant diseases detection to solve the classification of the disease. Machine learning and convolution networks have made an essential breakthrough in computer vision and many relevant theoretical and applied achievements have been reported [5]. Because CNNs can extract features consistently and straight from the input images, thereby avoiding complex pre-processing on images, they have been a research hotspot in object detection [26]. CNN-based model in the training process, natural disease leaf images are processed to generate sufficient training images via data augmentation technology [24]. A deep convolution neural network is employed for the real-time detection of leaf diseases. The proposed deep-learning-based approach can automatically identify the discriminative features of the diseased leaf images and detect the leaf diseases with high accuracy. A deep convolution neural network is employed for the real-time detection of curry leaf diseases [25].

\section{LITERATURE REVIEW}

The major role of image processing of plant diseases detection, there are several image processing techniques.
The mostly used algorithm for identifying the image processing of plant diseases detection is " $\mathrm{K}$-means Clustering" algorithm [17].

X. Zhang, Y. Qiao, F. Meng, C. Fan, and M. Zhang, "Identification of maize leaf diseases using better deep convolution neural networks," IEEE Access, vol. 6, pp. 30370-30377, 2018.

In the field of agricultural info, the automated identification and diagnosing of maize leaf diseases is highly desired. To enhance the identification accuracy of maize leaf diseases and scale back the quantity of network parameters, the improved Google internet and Cifar10 models supported deep learning area unit projected for plant disease recognition [25]. To improved models that accustomed train the leaf image and take a look at 9 kinds of maize leaf pictures area unit obtained by adjusting the parameters, dynamical the pooling combos, adding dropout operations and corrected linear measure functions, and reducing the quantity of classifiers. In addition, the quantity of parameters of the improved models is considerably smaller than that of the VGG and Alex internet structures. Throughout the popularity of eight types of maize leaf diseases, the Google internet model achieves a top-1 average identification accuracy of ninety eight.9\%, and also the Cifar10 model achieves Associate in Nursing average accuracy of ninety eight.8\% [4]. The improved ways are presumably improved the accuracy of maize leaf disease, and reduced the convergence iterations, which can effectively improve the model coaching and recognition with efficiency.

The requirements and procedure used are Dataset, Augmentation, Image pre-processing and labelling, convolution neural networks, hyper parameters, Equipment. In Dataset different images are collected 
during different periods of occurrence of maize leaf diseases. In augmentation, the more data is present which is to be learnt for more features it can obtain. The images collected will not be sufficient. To improve feature extraction and increase stability, the images in the database for the deep CNNs sorter are pre-processed before the model is trained. In Convolution neural networks cafe framework is used based on python language sketch especially for deep learning and CNNs- related algorithms. The improved cifar10 and Google Net models hyper parameters are compared with the original one [2]. Cafe framework, visual studio integrated development environment (IDE) and python language are used by them to train and test the complete model on a computer [24].

\section{S. P. Mohanty, D. P. Hughes, and M. Salathé, "Using} deep learning for image-based techniques for plant disease detection" Frontiers Plant Sci., vol. 7, p. 1419, Sep. 2016 [25].

Crop diseases are unit of significant threat to food security; however their fast identification remains tough in several components of the planet thanks to the dearth of the required infrastructure. The mix of accelerating international Smartphone penetration and up to date advances in pc vision created attainable by deep learning has finish the method for Smartphone-assisted wellness identification [12]. Deep convoulational neural networks (CNN) are with success applied in several numerous domains as samples of finish to finish learning. Neural networks give a mapping between input pictures of an unhealthy plant to output of crop wellness of plant. Employing a public dataset of fifty four, 306 pictures of unhealthy and healthy plant leaves collected underneath controlled conditions, we have a tendency to train a deep convolution neural network to spot fourteen crop species and twenty six diseases (or absence thereof). The trained model achieves
Associate in nursing accuracy of ninety nine.35\% on a held-out take a look at set, demonstrating the practicable of this approach [14]. Overall, the approach of coaching deep learning models on progressively massive and publically on the market image datasets presents a transparent path toward Smartphone-assisted crop unhealthy leaf identification on a colossal international scale.

Here they used plant village dataset where thousands of images of healthy and unhealthy plants are collected. To get a sense of how our approaches will perform on new unseen data, and also to keep a track of if any of our approaches are over fitting, we run all experiments across a whole range of train-test splits [3]. They evaluated the applicability of deep convolution neural networks for classification problem. Here they focused on two popular architectures, namely Alex Net and Google Net which were designed in the context of the "large scale visual recognition challenge" (ILSVRC). "They analyse the completion of both these architectures on the plant's database by training the patterns of image from scratch in one case, and then by adapting before trained patterns o f image using transfer learning'. Choices of datasets type are Colour, Gray Scale, and Leaf Segmented [13] [16].

After receiving the image that has diseases, the algorithm performs the image processing by converting the image from RGB colour space to HIS colour space. The colour and symptom of the diseases are be analysed by colour matrix and gray level cooccurrence matrix. The analysed the input to the Support Vector Machine (SVM) for identification and the identifying the plant diseases [10].

While the RGB image is used identifying the plant diseases. After applying the $\mathrm{k}$-means clustering algorithm the pixels of the image is identified by the method is called Otsu method and also varying 
threshold value. For the feature extraction colour cooccurrence method is used. RGB image converted into the HSI colour translation. From the texture statistics the "Spatial Gray-level Dependence Matrices (SGDM) is generated and using "Gray Level Co-occurrence Matrix (GLCM) from that the feature is analysed [10].

\begin{tabular}{|l|l|l|l|}
\hline Algorithm & Plant Disease & Dataset & $\begin{array}{l}\text { Accuracy } \\
\text { Occurred }\end{array}$ \\
\hline $\begin{array}{l}\text { Artificial } \\
\text { neural } \\
\text { network. }\end{array}$ & $\begin{array}{l}\text { Dermatological } \\
\text { Disease. }\end{array}$ & $\begin{array}{l}\text { Firstly } \\
\text { collected } \\
\text { dataset is25 }\end{array}$ & $99.35 \%$ \\
\hline Fulsunge & $\begin{array}{l}\text { Image } \\
\text { Processing } \\
\text { Using } \\
\text { MATLAB. }\end{array}$ & $\begin{array}{l}\text { Firstly } \\
\text { collected 37 } \\
\text { and } \\
\text { didn't } \\
\text { provide } \\
\text { validation }\end{array}$ & $86.07 \%$ \\
\hline $\begin{array}{l}\text { Soft } \\
\text { computing } \\
\text { techniques. }\end{array}$ & Pepper plants. & $\begin{array}{l}\text { Collected } \\
15 \text { first and } \\
\text { are un } \\
\text { valid }\end{array}$ & $97.1 \%$ \\
\hline $\begin{array}{l}\text { Back } \\
\text { propagation } \\
\text { algorithm }\end{array}$ & $\begin{array}{l}\text { Groundnut } \\
\text { leaf. }\end{array}$ & $\begin{array}{l}\text { Collected } \\
12 \\
\text { diseases leaf }\end{array}$ & $93.12 \%$ \\
\hline $\begin{array}{l}\text { Pattern } \\
\text { Recognition }\end{array}$ & Rice Disease. & $\begin{array}{l}\text { Collected } \\
17 \text { and 12 } \\
\text { unidentified }\end{array}$ & $73.07 \%$ \\
\hline
\end{tabular}

\section{Steps of Disease Detection:}

The method or steps of Plant diseases detection are five types of methods or steps. They are

1. Image Acquisition

2. Image Pre-processing

3. Image segmentation

4. Feature Extraction in image

5. Detection and Classification of plant diseases

These methods are mentioned in detail. In below

\section{Image Acquisition}

The image of the plant leaf and stem is taken or captured by camera and mobile phone. This image format can be .bmp, .jpg, .gif as input source of image plant diseases. The images can take from web resources from different types of plant diseases from images of plant diseases [10].

The database of image is completely depends on the application system of the images processing. The image diseases or plant diseases are should be in database of application system database from better efficiency of the spotting of plant diseases for application system by detecting the disease of plant by detection system.

The application system of database is responsible for the solution or remedies of disease to defend the apparatus of the disease that occurs to the crop. By the identifying the plant diseases of plant the solution to solve the plant diseases and identifying the plant diseases is should be in the application system database to identification of the plant diseases. The image database is better effectively to detect the disease of plant by detection systems.

\section{Image Pre-processing}

The image pre-processing is improves the image data is distortion of unwillingly data of suppresses in the image or enhances some of the image features for further processing, like geometric transformation of image (rotation, scaling) are classified among the preprocessing methods [8].

There are three types of image pre-processing. They are

- Pixel brightness transformation/ brightness correction.

- Geometric transformations.

- Image restoration. 
Image Pre-processing methods use the redundancy in images.

Distortion pixel can be restored as enhance of the average of image pixels.

Knowledge of the properties of the image acquisition deceives, and conditions under image were obtained.

Knowledge of the object of searched in the image, which may simply the pre-processing considerable.

If knowledge about of objects is not available, it can be estimated during the process [24].

\section{Pixel brightness transformation}

- Brightness transformation modifies or enhances pixel brightness. The transformation depends on the properties of pixels.

- Brightness correction :-

- Consider original brightness.

- Pixels are positioning in the image.

- Gray scale transformation

- Change brightness with or without positioning in the image [10].

- The image of the pixel depends on the brightness of the image and intensity of the image.

\section{Geometric transformations}

- Geometric transformations permit the geometrical distortion of the image that captured this will occurs in image [24].

- The geometrical of the image is calculated by mapping of the co-ordinators of the input image pixels to the point in the output image pixels.

- The output of the image co-ordinators of the original image be computed as continuous nature of the image in position does not match the necessarily of the digital grid in the image after the transform [15].
- The input image transforms into the output image by coordinating the co-ordinators Estimating or matching in same position the image is considered as the estimated image.

\section{Image restoration}

Image restoration is the operation or aim of image taking a corrupt/noise image and estimating the cleaning, original image corruption comes in many forms like motion blur, noise and camera miss-focus.

- Image restoration attempts to restore images that have been corrupted, degraded images.

- The clearance of the image of the noise and blurriness of the image is done by the image preprocessing method [18].

- Restoration of the image disorientated image can be restoring by the image restoration process.

- Encrypt of the image can be also done by the image restoration process.

- This process can be done by the some technical component of the image in pixels and the brightness, intensity of the image.

The image pre-processing techniques are done by these methods to the image to identify by the colour, co-ordinators format like etc.

\section{Image segmentation}

The goal of image segmentation is to simplify and/or change the represents of a picture into something that's deeper and simple to research. Segmentation is crucial stage of the image recognition system, because it extracts the objects of our interest, for further processing like description or recognition.

These papers propose an efficient procedure to pull out the leaf region and count the quantity of leaves in automated images of plant. The plant image examination plays a big role in practical and highyielding agriculture. It's wont to record the 
production of plant, crop, chlorophyll fluorescence, plant width and plant tallness, leaf area, etc. frequently and accurately.

Segmentation supported Clustering. Divides image pixels into homogeneous clusters. Segmentation techniques are wont to isolate the specified object from the image so as to perform analysis of the thing. Edge based and threshold techniques are usually with gray scale image of plant leaves and area Based, lot of data points in a group and classical algorithm segmentation technique used with colour of images of plant leaves [17]. The goal of segmentation is to regroup and furthermore change in the presentation of a picture into something that's more significant and fewer demanding to review. The after effect of picture pixel separation is an appointment of areas that each one thing considered cover the entire picture, where every pixel during a region is relative regarding some trademark or registered property, for instance, colour, intensity, or texture. We proposed reviews the varied segmentation techniques like Edge Based, Threshold, Region Based, Clustering and Watershed segmentation utilized in leaves analysis.

Machines are often taught to interpret images an equivalent way our brains do and to research those images far more thoroughly than we will. When applied to image processing, AI (AI) can power face recognition and authentic functionality for ensuring security publicly places, detecting and recognizing objects and patterns in images and videos, and so on [4].

In this article, we mention digital image processing and therefore the role of $\mathrm{AI}$ in it. We describe some AI-based image processing tools and techniques you'll use for developing intelligent applications. We also take a glance at the foremost popular neural network models used for various image processing task [20].

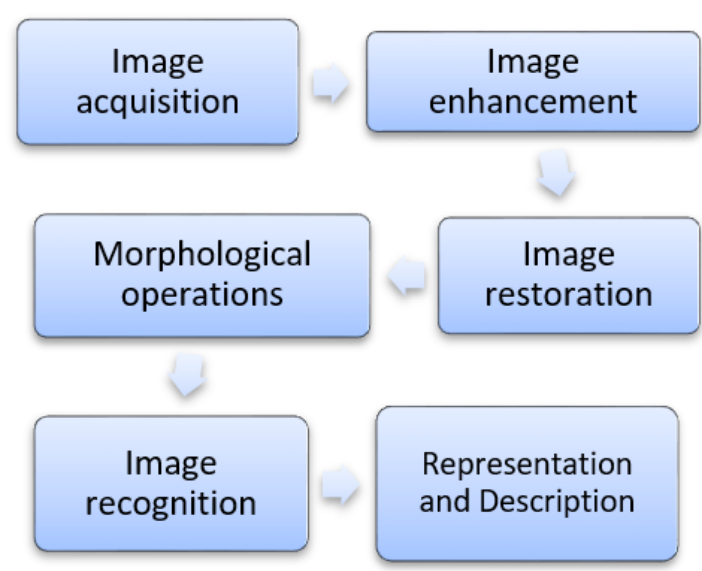

Fig.1. Key Phases of Digital Image Processing

- Analog image processing is employed for processing physical photographs, printouts, and other hard copies of images.

- Digital image processing is employed for manipulating digital images with the assistance of computer algorithms [24].

In most cases, the input is a picture. For analog image process, the output is normally related to picture. For automated image processing, however the output could also be picture information related to that image, like data on features, characteristics, bounding boxes, or masks.

Digital Image processing involves eight key points:

1. Image acquisition is that the process of capturing a picture with a device (such as a camera) and change into a possible entity (for example, a digital image file). Well liked image acquisition procedure is scraping.

At Apriority, we've created many image acquisition tools to assist our clients collect high-quality database for training neural network patterns.

2. Image enhancement improves the standard of a picture so as to extract hidden information from additional processing. 
3. Image restoration also improves the standard of a picture, mostly by removing possible corruptions so as to urge a cleaner version. This process is predicated totally on prospect and statistical models and may be wont to get obviate blur, noise, missing pixels, camera miss-focus, watermarks, and other corruptions which will negatively condition the instructions of a neural network [18].

4. Colour image processing includes the processing of collared images and different colour spaces. Counting on the image type, we will mention pseudo colour processing (when colours are assigned greyscale values) or RGB advancing (for images obtained with a full-colour sensor) [16].

5. Compression and decompression leave changing the dimensions and determination of a picture. Compression is liable for reducing the dimensions and determination, while decompression is employed for restoring a picture to its initial dimensions and determination

6. Morphological processing describes the forms and systemic of the entities in a picture. Morphological processing techniques are often used when generate the database for training AI models. Especially, morphological analysis and processing are often applied at the annotation stage, once you describe what you would like your AI model to observe or recognize

7. Image recognition is that the process of identifying specific features of particular objects in a picture. Image recognition with AI repeatedly uses such procedures for object detection, visual perception, and segmentation.

8. Representation and outline is that the process of picture and represent processed data. AI systems are planned to figure as efficiently as possible.

The output of an AI system seems like a collection of numbers and values that represent the knowledge the AI model was trained to detect.

\section{Feature Extraction:}

The feature extraction of the plant diseases detection in plant the major role is based on the "colour, texture, shape, edges of the leaf and also morphology can be used. The researchers are using texture features for detection of plant diseases [16] [5].

- Method of feature extraction "Gray-level Cooccurrence Matrix (GLCM).

- Colour Co-occurrence method.

- Spatial Gray-level Dependence Matrix.

- Histogram based feature extraction.

The GLCM method is a statistic method for texture classification. This above method is basically to identify the plant diseases. In this methods the detection of the plant diseases depends on the

- $\quad$ Shape feature extraction

- Colour feature extraction

- Texture feature extraction

\section{Shape feature extraction:}

The shape-oriented feature extraction like area, colour axis length or minor axis length, eccentricity, solidity and perimeter are calculated from this shape of feature extraction. The feature of image is taken to extract the disease [23].

\section{Texture feature extraction:}

Texture oriented feature extraction like contrast, correlation, energy, homogeneity and mean of the leaf or stem images of plant diseases. These features are taken to extract the diseases in plant leaf. Finally the variation of the pixel and the near to pixels are calculated [5]. 


\section{Colour feature extraction:}

Colour oriented feature extraction like concern with translation, scaling and rotation colour of feature extraction have a unique way of showing the image representing the image.

This initiative process of feature extraction of the image is analysing affected and unaffected areas of the leaf in a plant image. Then the image segment or analyzed and the name of the plant diseases identified. The image processing of the plant diseases detection provides the solution of the diseases and the percentage of the affected area in leaf and its surroundings region [16].

\section{Classification:}

The classification is done by two methods. They are

\section{Artificial Neural Network (ANN) :}

After classify the learning database image. Neurons in ANN are feature vector of the neural network. The weighted sum of the image input is the sum of the image output of the neuron in the function [1].

\section{Back propagation neural network (BPNN) :}

This BPNN is used in a recurrent the neural network. Once trained, the neural network weights are permanent and the neural network can be used to compute the output values of the query of the image which do not exist in the learning database [2].

After getting the weight of the learning database the testing of the neural network of the query image have done. The testing query of the image the neural network techniques used [6].

Completing the feature extraction of the plant diseases techniques, the neural network id used to [6].

The neural network techniques are
- $\quad$ Learning and testing

- Image database and input image

- Pre-processing and pre-processing

- Segmentation

- Feature extraction

- Weight adjustment

- Classification

- Comparison

- Diagnosis

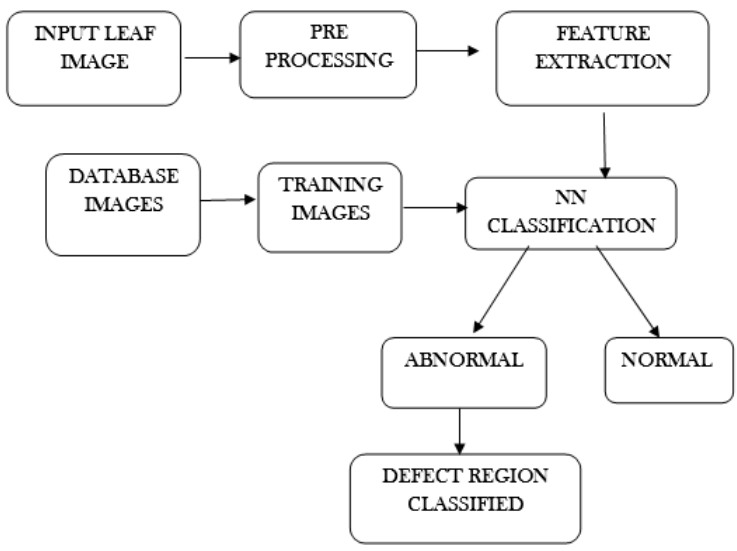

Fig. 2. Classification of the image processing [7]

\section{K-means Clustering algorithm:}

The K-means Clustering algorithm is used for classification of the objects based upon the k-clusters or set of characteristic into k-number of groups. The classification of objects is done by the minizing the cluster into sum of square of the space in the middle of the objects and the clusters [17].

\section{Algorithm:}

1. Pick centre of the $\mathrm{k}$ cluster, either randomly or based on the some heuristic.

2. Assign the each pixel in to cluster that minimize the distance in the middle of the pixel and the clusters. 
3. Again repeat the clusters by averaging all the pixels to the cluster. Repeat the steps of the 2 and 3 until convergence is obtained.

\section{Working of image processing by user case diagram:}

The image processing is the method which is using to detect the plant infection detection by the image of the plant infection and the solving or remedies of the diseases is provided by to detected diseases which are identified diseases.

By the given below diagram the image processing method is done.

- First the image is captured by the user.

- Then the image is uploaded to sever to identify the disease. This is called image acquisition.

- The second step is the image pre-processing of the image uploaded by the user to sever and it will do some specification to the image.

- Then the image segmentation is done to the image of the detection of the plant diseases by the input to output values of the image

- After that the feature extraction of the image is done to image from classification of the diseases.

- Classification is done by the ANN and BPNN from neural network weight of the classify the amount of the input is uniform to output of the image then the values are same the disease is Diagnosis and comparison is done by the database of the image to original image[1][2].

- Then training images says the disease is detected or not. If it is detected then the disease name what are precautions and solutions to the disease.

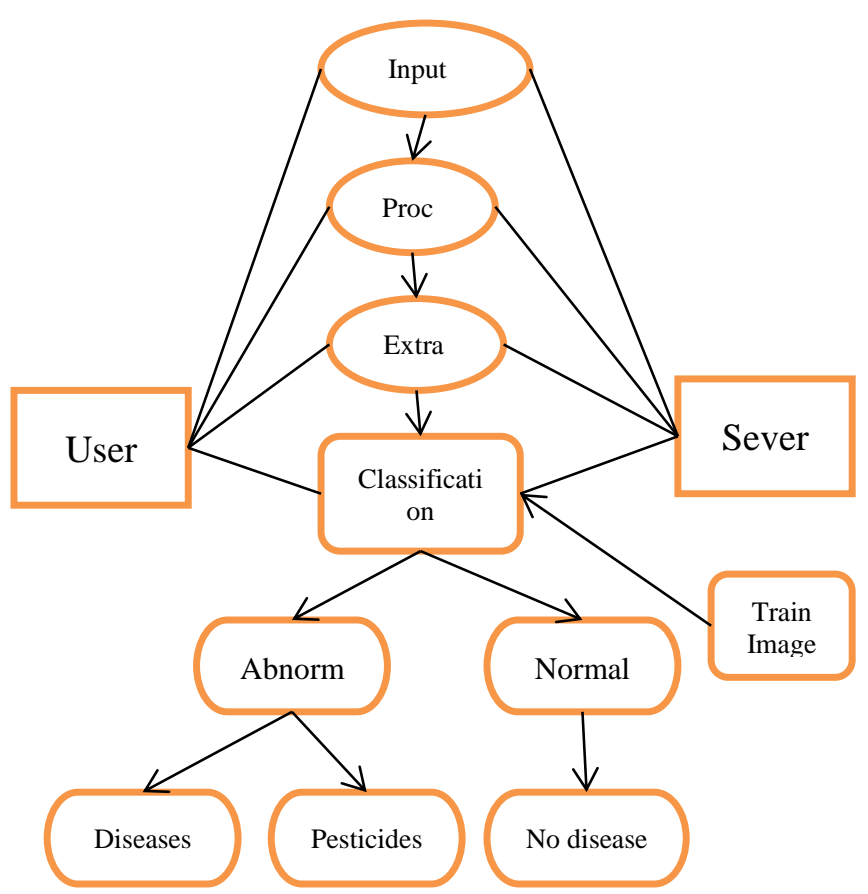

Fig.3. user case diagram in classification of the image processing

\section{CONCLUSION}

This research paper represents the identification of the plant diseases or leaf diseases are main purpose of the deep learning using image pre-processing. Various types of techniques are utilizing to identify the plant diseases. K-means Clustering, Neural network are used in image processing to detect the disease and the infected region of the leaf is segmented and analyzed.

The feature extraction of the plant diseases detection techniques helps to extract the infected region in the leaf or plant to identify the diseases. The major role of this technology is depends on the software and the database of the application system. We use the MALAB in python programming for the image processing techniques are more efficient. 


\section{REFERENCES}

[1]. Rahat Yasir, Md. Ashiqur Rahman, "Dermatological Disease detection using imame procession and artificial neural network". ICECE, International Conference on Pp. 687690, IEEE, 2014.

[2]. SandeshRaunt, AmitFulsunge "Plant Disease Detection of Image Processing Using MATLAB” IJIRSET Vol. 6, Issue 6, June 2017

[3]. Barbedo, J.G.A., 'A review on the main challenges in computerized plant disease identification based on visible range image ', 2016, Bio system Engineering Volume 144.

[4]. Santanu Phadikar and Jaya Sil, "Rice Disease Identification Using Pattern Recognition ", Processing of 11th International Congress on Computer and Information Technology (ICCIT 2008) 25-27 December, 2008, Khulna, Bangladesh.

[5]. Dr.K.Thangaduri, K.Padmavathi, "Computer Vision image improvement for Plant Leaves Disease Detection", 2014 World Conference on Computing and Communication Technologies.

[6]. M.Ramakirshna, Sahaya Anselin Nisha, "Groundnut leaf disease recognition and categorization by using back propagation algorithm", In Communication and Signal Processing (ICCSP), International Conference on, pp. 0964-0968, IEEE, 2015.

[7]. Dhakate, Mrunmayee and A.B. Ingole. "Diagnosis of pomegranate plant disease using neural network", In NCVPRIPG, Fifth National conference on, pp. 1-4, IEEE, 2015.

[8]. Shan, J.P. Prajapati, H.B. and Dabhi, V.K., "A Survey on recognition and categorization of rice plant disease". In Current Trends in Advanced Computing (ICCTAC), International Conference on, pp. 1-8, IEEE, 2016.

[9]. Francis, Jobin, and B.K. Anoop, "Identification of leaf disease in pepper plants using soft computing techniques", In Emerging SensorS and Smart Systems (ICEDSS), Conference on, pp. 168-173, IEEE, 2016.

[10]. K. Padmavathi, and K. Thangadurai, "Implementation of RGB and Gray scale images In plant leaves disease recognition -relative Study," Indian J. of Sci. and Tech., vol. 9, pp. 16, Feb. 2016.

[11]. Kiran R. Gavhale, and U. Gawande, "An Survey of the Research on Plant Leaves Disease recognition using Image Processing Techniques," IOSR J. of Computer Eng. (IOSRJCE), Vol. 16, PP 10-16, Jan. 2014.

[12]. Monica Jhuria, Ashwani Kumar, and RushikeshBorse, "Image Processing For Smart Farming: Detection of Disease and Fruit Grading”, Proceedings of the 2013 IEEE Second International Conference on Image Information Processing, 2013

[13]. K. Elangovan, S. Nalini "Plant Disease Classification Using Image Segmentation and SVM Techniques" IJCIRV ISSN 0973-1873 Vol. 13, 2017

[14]. H. Al-Hiary, S. Bani-Ahmad, M. Reyalat, M. Braik and Z. ALRahamneh, "Fast and Accurate Detection and Classification of Plant Diseases", International Journal of Computer Applications (0975 - 8887) Volume 17- No.1, March 2011

[15]. Chunxia Zhang, Xiuqing Wang, Xudong Li, "Design of Monitoring and Control Plant Disease System Based on DSP\&FPGA", 2010 Second International Conference on Networks Security, Wireless Communications and Trusted Computing.

[16]. A. Meunkaewjinda, P. Kumsawat, K. Attakitmongcol and A. Srikaew, "Grape leaf disease detection from color imagery using hybrid intelligent system", Proceedings of ECTI-CON 2008.

[17]. Trimi Neha Tete, Sushma Kamlu, "Detection of Plant Disease Using Threshold, K-Mean Cluster 
and ANN Algorithm", 2014 World Congress on Computing and Communication Technologies.

[18]. Sachin D.Khirade, A.B. Patil "Plant Disease Detection using image processing. International Conference on Computing Communication Control and Automation. "2017, 2nd International Conference for Convergence in Technology

[19]. Taohidul Islam, Manish Sah, Sudipto Baral, Rudra Roy Choudhury "A faster technique on rice disease detection using image processing of affected area in agro field", 2018 Proceedings of the 2nd International Conference on Inventive Communication and Computational Technologies.

[20]. Varsha P. Gaikwad, Dr. Vijaya Musande "Wheat Disease Detection Using Image Processing" IEEE, Conference 2017.K. Elissa, "Title of paper Know", Unpublished.

[21]. Bashish, D.A., Braik, M., Ahmad, S.B., 'A Framework for Detection and Classification of Plant Leaf and Stem Diseases', International Conference on Signal and Image Processing, pp. 113-118, 2010.

[22]. Punajari, J.D., Yakkundimath, R., Byadgi, A.S., 'Image Processing Based Detection of Fungal Diseases In Plants', International Conference on Information and Communication Technologies, Volume 46, pp. 1802-1808, 2015.

[23]. Omrani, E., Khoshnevisan, B., Shamshirband, S., Saboohi, H., Anuar, N.B., Nasir, M.H.N., 'Potential of radial basis function based support vector regression for apple disease detection', Journal of Measurement, pp. 233-252, 2014.

[24]. Gharge, S., Singh, P., 'Image Processing for Soybean Disease Classification and Severity Estimation', Emerging Research in Computing, Information, Communication and Applications, pp. 493-500, 2016

[25]. S. P. Mohanty, D. P. Hughes, and M. Salathé, "Using deep learning for image-based plant disease detection," Frontiers Plant Sci., vol. 7, p. 1419, Sep. 2016.

[26]. X. Zhang, Y. Qiao, F. Meng, C. Fan, and M. Zhang, "Identification of maize leaf diseases using improved deep convolution neural networks," IEEE Access, vol. 6, pp. 3037030377, 2018.

\section{Cite this article as :}

Vempati Ramsanthosh, Anati Sai Laxmi, Chepuri Sai Abhinay, Vadepally Santosh, Vybhav Kothareddy, Shivali Chopra, "Review and Further Prospects of Plant Disease Detection Using Machine Learning", International Journal of Scientific Research in Computer Science, Engineering and Information Technology (IJSRCSEIT), ISSN : 2456-3307, Volume 7 Issue 3, pp. 105-115, May-June 2021. Available at doi : https://doi.org/10.32628/CSEIT217324 Journal URL : https://ijsrcseit.com/CSEIT217324 